\title{
Bobbing of Oxysterols : Molecular Mechanism for Translocation of Tail-Oxidized Sterols through Biological Membranes
}

\section{Kulig, Waldemar}

2018-03-01

Kulig , W , Mikkolainen , H, Olzynska , A , Jurkiewicz, P, Cwiklik , L , Hof , M , Vattulainen , I , Jungwirth , P \& Rog , T 2018 , ' Bobbing of Oxysterols : Molecular Mechanism for

Translocation of Tail-Oxidized Sterols through Biological Membranes ' , Journal of Physical Chemistry Letters , vol. 9 , no. 5 , pp. 1118-1123 . https://doi.org/10.1021/acs.jpclett.8b00211

http://hdl.handle.net/10138/322175

https://doi.org/10.1021/acs.jpclett.8b00211

acceptedVersion

Downloaded from Helda, University of Helsinki institutional repository.

This is an electronic reprint of the original article.

This reprint may differ from the original in pagination and typographic detail.

Please cite the original version. 


\section{Bobbing of Oxysterols: Molecular Mechanism for Translocation of Tail-Oxidized Sterols through Biological Membranes}

Waldemar Kulig, a,b Heikki Mikkolainen, ${ }^{\mathrm{b}}$ Agnieszka Olżyńska, ${ }^{\mathrm{c}}$ Piotr Jurkiewicz, ${ }^{\mathrm{c}}$ Lukasz Cwiklik, ${ }^{c, d}$ Martin Hof,c Ilpo Vattulainen, ${ }^{a, b, e}$ Pavel Jungwirth, ${ }^{d^{*}}$ Tomasz Rog, ${ }^{a, b},{ }^{*}$

aDepartment of Physics, P. O. Box 64, FI-00014 University of Helsinki, Finland

bLaboratory of Physics, Tampere University of Technology, P. O. Box 692, Fl-33101, Finland

cJ. Heyrovský Institute of Physical Chemistry, Academy of Sciences of the Czech Republic, v.v.i., Dolejškova 3, 18223 Prague,Czech Republic

${ }^{d}$ Institute of Organic Chemistry and Biochemistry, Academy of Sciences of the Czech Republic, 16610, Prague, Czech Republic

eMEMPHYS- Center for Biomembrane Physics

*E-mail: tomasz.rog@gmail.com, pavel.jungwirth@uochb.cas.cz 


\section{ABSTRACT}

Translocation of sterols between cellular membrane leaflets is of key importance in membrane organization, dynamics, and signaling. We present a novel translocation mechanism that differs in a unique manner from the established ones. The bobbing mechanism identified here is demonstrated for tail-oxidized sterols, but is expected to be viable for any molecule containing two polar centers at the opposite sides of the molecule. The mechanism renders translocation across a lipid membrane possible without a change in molecular orientation. For tail-oxidized sterols, the bobbing mechanism provides an exceptionally facile means to translocate these signaling molecules across membrane structures and may thus represent an important pathway in the course of their biological action.

\section{TOC IMAGE:}

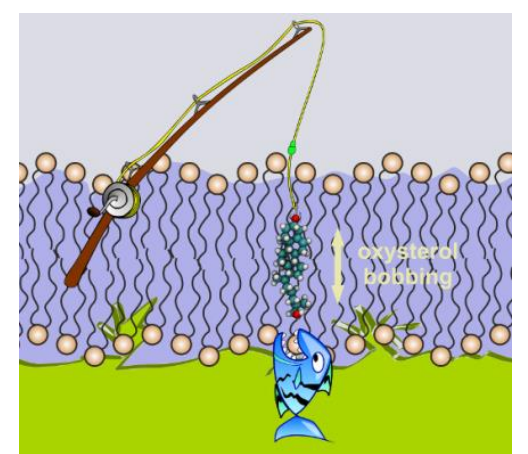


Biological membranes are highly diverse macromolecular aggregates composed of numerous lipid species and proteins, each having specific functions. The function of lipids depends to a large extent on their distribution in the membranes. As a matter of fact, an asymmetric transmembrane distribution of lipids is a rule rather than an exception. This holds for essentially all lipid species in numerous membrane types, ${ }^{1,2}$ being actively maintained by cells via a set of enzymes called flippases, floppases, and scramblases translocating phospholipids between the two membrane leaflets. ${ }^{3-5}$ This asymmetry is of vast importance not only for membrane properties per se, but also for transmembrane signaling mechanisms. For example, phosphatidylserine under normal conditions functions in the cytosolic leaflet of a plasma membrane, but becomes a messenger of cell death when translated to the extracellular cell surface.

Oxidative modification of cholesterol $(\mathrm{CHOL})$ can occur either by an attack by reactive oxygen species (ROS) or via relatively well-controlled cellular processes involving different enzymes, ${ }^{6-7}$ mostly related to the cytochrome P450 family. While ROS generate ring-oxidized sterols, the enzymatic process mostly leads to oxidation of the sterol tail. Within these distinct mechanisms, formation of oxysterols with two polar moieties decorating two different sides of the steroid system thus takes place.

Oxysterols are considered as crucial players in many biological processes despite their relatively small concentrations in human tissues. Their biological functions are comprehensive, including, e.g., regulation of gene expression, ${ }^{8-9}$ roles as atherogenic agents, ${ }^{10-12}$ modulators of cell membrane permeability ${ }^{13-16}$ and metabolic intermediates, ${ }^{7,} 17$ and functions as ligands in cellular signaling. ${ }^{18-20}$

The tail-oxidized oxysterols (such as 24-, 25-, and 27-hydroxycholesterol) that we focus on in this work act mostly as signaling molecules to bring up the excess of cholesterol in the cells. They regulate cholesterol homeostasis ${ }^{21-23}$ by, e.g., suppressing the activity of HMG-coenzyme A reductase (the rate-limiting enzyme in the cholesterol synthesis pathway) and stimulating cholesterol esterification with its subsequent storage in lipid droplets. ${ }^{24-26}$

To fulfill the above-mentioned functions, tail-oxidized sterols need to move rapidly between different cellular organelles such as the endoplasmic reticulum and plasma membrane. To do so, their translocation across membrane structures should also be fast. Here, we show how tail-oxidized oxysterols carry out membrane translocation through a unique molecular mechanism not found for any other lipid prior to this study.

We employed unbiased molecular dynamics (MD) simulations combined with free energy calculations (umbrella sampling), ${ }^{27}$ using an analogous simulation setup as in our previous studies. ${ }^{14}$ The oxysterols considered here included $7 \beta$-hydroxycholesterol $(7 \beta-\mathrm{OH}-\mathrm{CHOL})$, where oxidation takes place in the sterol ring by modifying $\mathrm{CHOL}$ structure by an additional hydroxyl group, and 27hydroxycholesterol (27-OH-CHOL), where oxidization modifies the structure of the short terminal tail (Figure 1C). These two representative cases cover the typical structural modifications associated with oxysterols, i.e., oxidation due to free radicals modifying the sterol ring and enzymatic oxidation taking place in the sterol tail. This choice of a typical tail-oxidized sterol (27-OH-CHOL) vs. a ring-oxidized sterol $(7 \beta-\mathrm{OH}-\mathrm{CHOL})$ is sufficient to demonstrate the profound difference between these two classes of sterols. In addition, $\mathrm{CHOL}$ is studied as a control. The lipid membranes studied were comprised of $90 \mathrm{~mol} \%$ POPC and $10 \mathrm{~mol} \%$ of a sterol (i.e., $\mathrm{CHOL}, 7 \beta-\mathrm{OH}-\mathrm{CHOL}$, or $27-\mathrm{OH}-\mathrm{CHOL}$ ). Simulations were 
performed with the GROMACS simulation package ${ }^{28-29}$ using the all-atom OPLS-AA force field for lipids and sterols. ${ }^{14,30-31} \mathrm{~A}$ more extensive description of the methods is given in the Computational Methods section. Topologies of sterol molecules are included in the Supplementary Information.

First, we performed free energy calculations to quantify the translocation of $\mathrm{CHOL}$ across the bilayer. The obtained free energy profile shown in Figure $1 \mathrm{~A}$ (red line) is symmetric, demonstrating the existence of two equivalent free energy minima at a distance of about $1 \mathrm{~nm}$ from the bilayer center. Based on these positions, the free energy barrier for $\mathrm{CHOL}$ translocation between the two leaflets of the lipid bilayer is around $13.8 \mathrm{~kJ} / \mathrm{mol}$ (Figure 1A), in agreement with previous studies. ${ }^{32-34}$ In the positions identified as free energy minima, the cholesterol hydroxyl group accommodates at the membrane-water interface, the tilt of the cholesterol ring (for precise definition see Supplementary Information) being about $20-30^{\circ}$ with respect to membrane normal. This is further corroborated by the tilt angle distribution shown in Figure S2. However, during the translocation process the tilt of $\mathrm{CHOL}$ depends on its position along the membrane normal (Figure 1B). This is highlighted by an abrupt change around the membrane center, where $\mathrm{CHOL}$ turns upside down, demonstrating the established translocation mechanism in line with previous studies of $\mathrm{CHOL}$ translocation. ${ }^{32-34}$

For the ring-oxidized $7 \beta-\mathrm{OH}-\mathrm{CHOL}$, the calculations revealed a free energy profile (Figure $1 \mathrm{~A}$, green line) that is rather similar to that of $\mathrm{CHOL}$. However, the barrier for $7 \beta-\mathrm{OH}-\mathrm{CHOL}$ translocation is slightly larger, reflecting the presence of two polar groups in the $7 \beta-\mathrm{OH}-\mathrm{CHOL}$ structure (Figure $1 \mathrm{C}$ ). Like for $\mathrm{CHOL}$, the $7 \beta-\mathrm{OH}-\mathrm{CHOL}$ orientation changes again abruptly in the membrane center, in line with the established translocation mechanism (Figure 1B). The tilt angle distribution (see Figure S2) demonstrates that the most probable orientation of $7 \beta-\mathrm{OH}-\mathrm{CHOL}$ corresponds to the situation where sterol rings are tilted about $20-30^{\circ}$ with respect to membrane normal. A small artificial asymmetry in the free energy profile between the two bilayer leaflets, resulting in a difference of about $4 \mathrm{~kJ} / \mathrm{mol}$ in the positions of free energy minima (see Table 1), is due to a slightly different lipid composition between the two leaflets in the closest vicinity of the translocating sterol. This local asymmetry results from local movement of sterols and lipids within leaflets, with the average composition of both leaflets being identical.

Unbiased MD simulations carried out for POPC/CHOL and $\mathrm{POPC} / 7 \beta-\mathrm{OH}-\mathrm{CHOL}$ revealed no spontaneous translocation events during the simulation time of $1 \mu \mathrm{s}$. Taken together with the high viscosity of the membrane interior, this is consistent with the free energy barriers of $14-18 \mathrm{~kJ} / \mathrm{mol}$ (i.e., 6-8 $k_{\mathrm{B}} T$ ) observed for translocation of these two sterols (Figure $1 \mathrm{~A}$ ). 

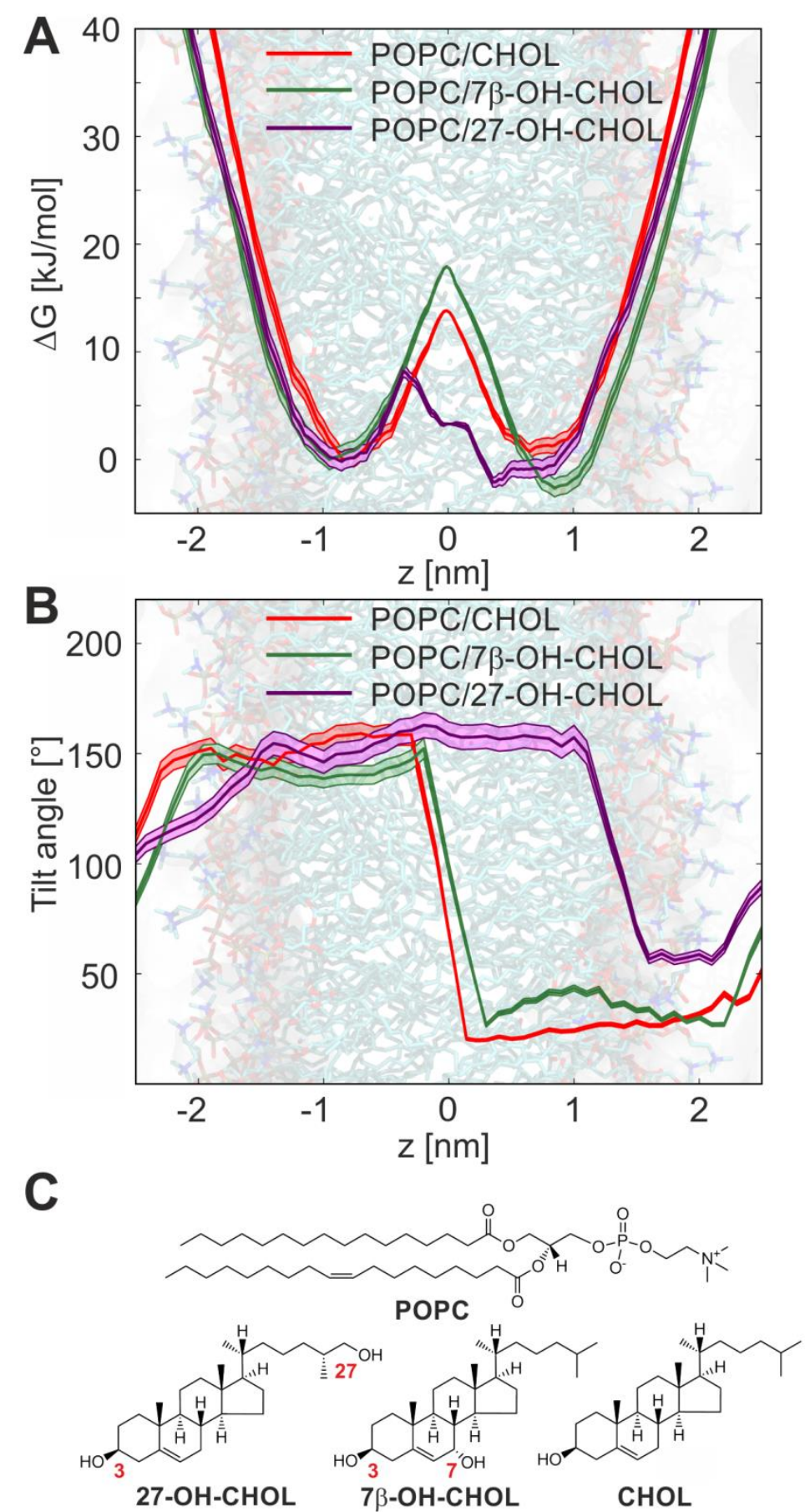

Figure 1. (A) Free energy profiles of $\mathrm{CHOL}$ (red), $27-\mathrm{OH}-\mathrm{CHOL}$ (purple), and $73-\mathrm{OH}-\mathrm{CHOL}$ (green). The transparent area around each curve shows the corresponding statistical error. When the $3 \beta$-hydroxyl group of 27-OH-CHOL faces water $(z>0)$, we call this the parallel orientation; when its 27-hydroxyl group faces water, we call this the antiparallel orientation. (B) Time evolution of the tilt angle (for definition see Supplementary Information) of each sterol molecule with respect to bilayer normal, $90^{\circ}$ representing a situation where the sterol molecule is perpendicular to the bilayer normal, while $0^{\circ}$ and $180^{\circ}$ correspond to the situations where the sterol molecule is parallel to the bilayer normal. (C) Molecular structures of sterols and lipids studied. The $z$ coordinate represents the position of the sterol's centre of mass. 
The situation changes quite radically when the dynamics of the tail-oxidized $27-\mathrm{OH}-\mathrm{CHOL}$ is being considered (Movie S1, DOI: 10.5281/zenodo.1171345). In contrast to $\mathrm{CHOL}$ and $7 \beta-\mathrm{OH}-\mathrm{CHOL}$, the translocation of 27-OH-CHOL is a very frequent event. Namely, we observed translocations of 27$\mathrm{OH}-\mathrm{CHOL}$ taking place at a high rate of about $1 / 560$ ns per molecule (i.e., there were about 20 translocation events within 800 ns of unbiased simulation containing 14 sterol molecules). Even more intriguingly, the results revealed that the translocation mechanism is completely different from that observed for $\mathrm{CHOL}$ or $7 \beta-\mathrm{OH}-\mathrm{CHOL}$. No change of sterol orientation with respect to the bilayer normal was observed. What happens instead may be best described as "bobbing" of the sterol molecule, where 27-OH-CHOL moves back and forth along the membrane normal almost like a piston in a cylinder, thereby steadily moving across the bilayer without changing its orientation. The $27-\mathrm{OH}-\mathrm{CHOL}$ oxysterol (Figure 1C) moves from a position, where its $3 \beta$-hydroxyl group is exposed to the water phase and the 27-hydroxyl group is buried inside the lipid bilayer, to a new position, where the 27-hydroxyl group is exposed to the water phase and the $3 \beta$-hydroxyl group is buried inside the bilayer core. The tilt angle distribution (see Figure S2) clearly shows that the most probable orientation of $27-\mathrm{OH}-\mathrm{CHOL}$ molecule is parallel to the bilayer normal.

Figure 2 shows snapshots from umbrella sampling simulations, illustrating the bobbing of 27$\mathrm{OH}-\mathrm{CHOL}$, as contrasted to the translocation with a flip of $7 \beta-\mathrm{OH}-\mathrm{CHOL}$ (see also Figure $\mathrm{S} 1$ in Supplementary Information). In the analysis, the bobbing of $27-\mathrm{OH}-\mathrm{CHOL}$ revealed local deformation with the bilayer becoming thinner as the hydroxyl groups (3ß-hydroxyl or 27-hydroxyl) became exposed to the membrane-water interface.

Table 1. The free energy barriers for sterol translocation between different leaflets of POPC bilayer.

\begin{tabular}{c|c}
\hline \hline System & Free energy barrier $(\mathrm{kJ} / \mathrm{mol})$ \\
\hline \hline $\mathrm{POPC} / \mathrm{CHOL}$ & $14 \pm 1$ \\
$\mathrm{POPC} / 7 \beta-\mathrm{OH}-\mathrm{CHOL}$ & $18 \pm 1$ \\
$\mathrm{POPC} / 27-\mathrm{OH}-\mathrm{CHOL}$ & $8 \pm 1 / 10 \pm 1^{*}$ \\
\hline \hline
\end{tabular}

* The free energy barrier from a parallel orientation with the $3 \beta$-hydroxyl group of $27-\mathrm{OH}-\mathrm{CHOL}$ facing water to an antiparallel setting with the 27-hydroxyl group facing water and that in the opposite direction.

Given that the translocation mechanism of $27-\mathrm{OH}-\mathrm{CHOL}$ is distinctly different from that of $\mathrm{CHOL}$ or $7 \beta-\mathrm{OH}-\mathrm{CHOL}$, one can expect that also the free energy barrier for the translocation of $27-\mathrm{OH}-$ $\mathrm{CHOL}$ is different compared to that of $\mathrm{CHOL}$ or $7 \beta-\mathrm{OH}-\mathrm{CHOL}$. This is indeed the case (Figure $1 \mathrm{~A}$, purple). The free energy barrier from a parallel orientation (the $3 \beta$-hydroxyl group of $27-\mathrm{OH}-\mathrm{CHOL}$ facing water) to an antiparallel setting (the 27-hydroxyl group facing water) is now reduced down to a value of about $8.2 \mathrm{~kJ} / \mathrm{mol}$ (i.e., roughly $3 k_{\mathrm{B}} T$ ), explaining also why bobbing was observed frequently in unbiased $\mathrm{MD}$ simulations (Movie S1, DOI: 10.5281/zenodo.1171345). In the opposite direction (from the antiparallel to the parallel orientation), the barrier is slightly higher, i.e., $10.3 \mathrm{~kJ} / \mathrm{mol}$ (about $4 k_{\mathrm{B}} T$ ).

The orientation of 27-OH-CHOL does not change on the timescale of the translocation process, which explains why the reported free energy profile is asymmetric: i.e., one does not need to consider here the much slower subsequent rotation of $27-\mathrm{OH}-\mathrm{CHOL}$ since, unlike for $\mathrm{CHOL}$ or $7 \beta-\mathrm{OH}-\mathrm{CHOL}$, such a rotation is not required for the translocation process. The two free energy minima are located at about $0.4 \mathrm{~nm}$ (parallel orientation) and $0.9 \mathrm{~nm}$ (antiparallel orientation) from the bilayer center, with the former being about 2-3 kJ/mol deeper than the latter (Figure 1A). The presence of two polar groups 
at the opposite ends of $27-\mathrm{OH}-\mathrm{CHOL}$ thus has a pronounced effect on the free energy profile, reducing the translocation free barrier to a value comparable to the energy of thermal fluctuations.

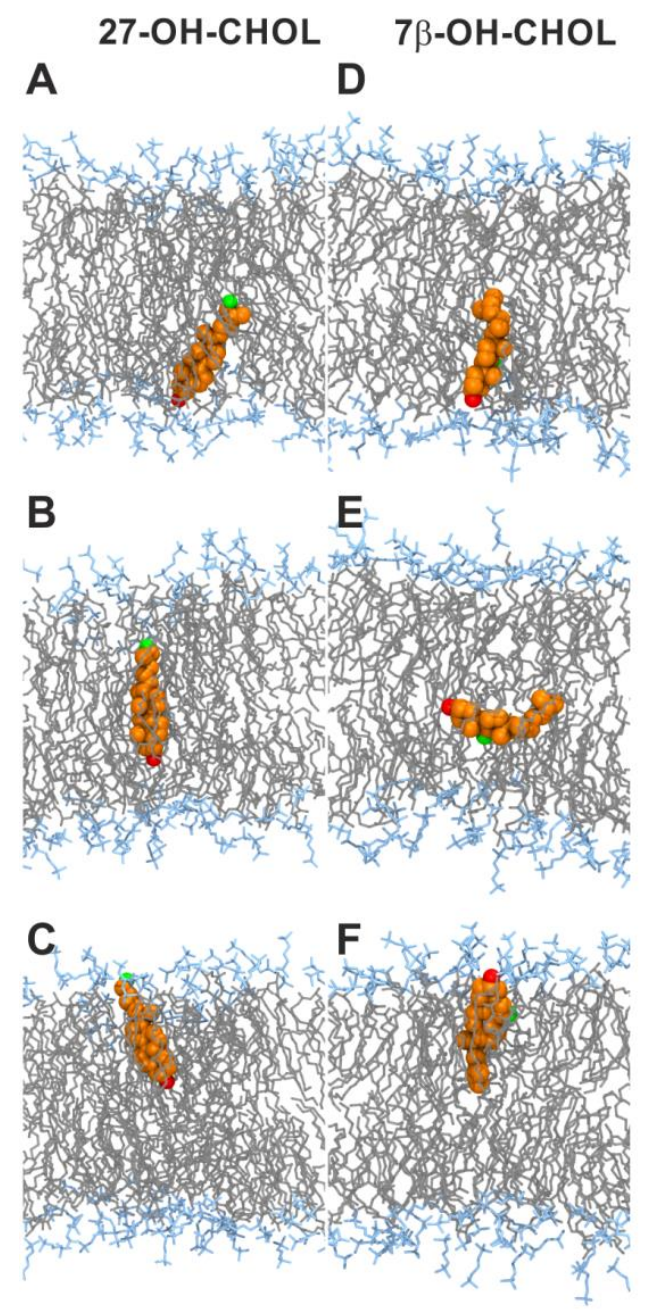

Figure 2. Snapshots of (A-C) 27-OH-CHOL and (D-F) $7 \beta-\mathrm{OH}-\mathrm{CHOL}$ oxysterols during their translocation processes across a membrane. Shown here are the $3 \beta$-hydroxyl group (red), the $7 \beta$ - and 27 -hydroxyl groups (green), the oxysterols' ring/tail structures (orange), the POPC headgroup (cyan), and lipid chains (grey).

While the bobbing mechanism has not been identified in previous studies, a similar mechanism was suggested as a possible explanation for the increase of bilayer permeability upon enrichment in $25-\mathrm{OH}-\mathrm{CHOL} .{ }^{35} \mathrm{~A}$ previous theoretical study ${ }^{33}$ using an implicit membrane model to evaluate the free energy landscape related to sterol translocation through a lipid bilayer also predicted the existence of two asymmetric minima, in agreement with the results presented here. However, the free energy barrier obtained in the present study is smaller than predicted previously, which is mainly due to formation of local membrane deformations facilitating bobbing. This effect simply could not be recovered in previous studies employing an implicit membrane model. ${ }^{33}$ Finally, our results point to the fact that the bobbing behavior of tail-oxidized sterols is not operational for $\mathrm{CHOL}$ or ring-oxidized sterols, which also decrease membrane permeability for water, gases, and small drug-like molecules. ${ }^{36-}$ 43 
To summarize, we identified and characterized a novel mechanism of molecular translocation through a lipid bilayer. Unlike the established flip-flop mechanism, ${ }^{33-34,44-46}$ in which a sterol molecule changes its orientation to accommodate its $3 \beta$-hydroxyl group at the membrane-water interface, the bobbing mechanism identified here involves translocation without a change in molecular orientation (which, nevertheless, may subsequently take place at longer timescales through a rotational motion in the interfacial region). The bobbing mechanism was observed here only for $27-\mathrm{OH}-\mathrm{CHOL}$, which contains an additional hydroxyl group located at the sterol tail. The unbiased simulations of POPC/7 $\beta$ $\mathrm{OH}-\mathrm{CHOL}$ and POPC/CHOL systems did not show any sign of bobbing due to the lack of polar group in the tail. It is plausible to assume that similar translocation events take place also with other molecular species having two polar centers at opposite ends of the molecule.

Our results suggest that bobbing is a frequent process occurring at the nanosecond time scale. Since tail-oxidized sterols act as signaling molecules, their dynamic properties are of crucial importance for cell function. It has been demonstrated ${ }^{47-48}$ that $25-\mathrm{OH}-\mathrm{CHOL}$ transfers rapidly from cell membranes to the endoplasmic reticulum with a rate that is a hundred times larger compared to that of $\mathrm{CHOL}$. Similarly, the transport rate of $24 \mathrm{~S}-$ and $27-\mathrm{OH}-\mathrm{CHOL}$ from erythrocytes to the plasma membrane is high, while similar transport of $4 \beta-\mathrm{OH}-\mathrm{CHOL}$ and $\mathrm{CHOL}$ is barely measurable. ${ }^{16}$ Bobbing may thus be seen as a potential mechanism facilitating rapid translocation of tail-oxidized oxysterols through cellular compartments towards receptors, often localized in the nucleus surrounded by two membranes.

\section{COMPUTATIONAL METHODS}

All-atom molecular dynamics (MD) simulations were performed for bilayers consisting of $90 \mathrm{~mol} \%$ of POPC and $10 \mathrm{~mol} \%$ of cholesterol $(\mathrm{CHOL}), 7 \beta-\mathrm{OH}-\mathrm{CHOL}$, or $27-\mathrm{OH}-\mathrm{CHOL}$ (denoted below as $\mathrm{POPC} / \mathrm{CHOL}, \mathrm{POPC} / 7 \beta-\mathrm{OH}-\mathrm{CHOL}$, and $\mathrm{POPC} / 27-\mathrm{OH}-\mathrm{CHOL}$, respectively). The initial structure of the POPC bilayer was created by arranging POPC molecules on an $8 \times 8$ grid, resulting in a bilayer consisting of two leaflets with 64 lipids each. Bilayers containing $10 \mathrm{~mol} \%$ of a sterol were constructed by randomly exchanging 14 lipid molecules by sterol molecules. The lipid composition of both leaflets was identical.

Each system was first energy minimized using the steepest descent algorithm and then equilibrated in the NpT ensemble for at least 50 ns. Finally, two sets of simulations were performed. The first set consisted of three unbiased simulations, each 1 microsecond long, where the last 800 ns were used for analysis. The second set consisted of three umbrella sampling simulations. The umbrella sampling simulations were performed in a manner, where a single sterol molecule was pulled from the membrane-water interface toward the bilayer centre. Prior to the umbrella sampling simulations, corresponding pulling simulations were performed in order to generate a series of configurations for umbrella sampling. In the pulling simulations, the investigated molecules were pulled along the $z$ axis and the center of mass (COM) of the sterol molecule was used as the pulled group. The force constant applied to the pulled sterol molecule was $500 \mathrm{~kJ} \mathrm{~mol}^{-1} \mathrm{~nm}^{-2}$ and the pull rate was $0.001 \mathrm{~nm} \mathrm{~ns}^{-1}$. After pulling simulations, umbrella sampling was performed with a total of 71 windows. The initial configurations for umbrella windows were selected randomly at $0.1 \mathrm{~nm}$ intervals. Each window was simulated for $100 \mathrm{~ns}$, where the first $40 \mathrm{~ns}$ were used for equilibration. In each umbrella window, the pulled molecules were restrained using a harmonic potential with a force constant of $3000 \mathrm{~kJ} \mathrm{~mol}^{-1} \mathrm{~nm}^{-}$ 
2. The restrains were applied only along the reaction coordinate (i.e., along the normal to the bilayer surface), while the pulled molecules were free to move in other directions. Additional computational details are given in the Supplementary Information.

\section{ACKNOWLEDGEMENTS}

For financial support, we thank the Academy of Finland (the Academy of Finland Distinguished Professor program (grant no. 263410 (WK)) and the Center of Excellence program (grant no. 307415 (IV))), the European Research Council (Advanced Grant CROWDED-PRO-LIPIDS, grant no. 290974 (IV)), the Czech Science Foundation (grant no. P208/12/G016 (PJ and MH)), and the European Regional Development Fund OP RDE (project Chemical biology for drugging undruggable targets ChemBioDrug no. CZ.02.1.01/0.0/0.0/16_019/0000729 (PJ)). CSC-IT Centre for Science (Espoo, Finland) and the Finnish Grid and Cloud Infrastructure (persistent identifier urn:nbn:fi:research-infras-2016072533) are acknowledged for excellent computational resources.

\section{SUPPORTING INFORMATION}

Extended computational details, additional results, molecular topologies and structure files of oxysterols, and movie of the $27-\mathrm{OH}-\mathrm{CHOL}$ bobbing.

\section{References:}

1. van Meer, G.; Voelker, D. R.; Feigenson, G. W. Membrane lipids: where they are and how they behave. Nature Reviews Molecular Cell Biology 2008, 9 (2), 112-124. 2. Takamori, S.; Holt, M.; Stenius, K.; Lemke, E. A.; Grønborg, M.; Riedel, D.; Urlaub, H.; Schenck, S.; Brügger, B.; Ringler, P. Molecular anatomy of a trafficking organelle. Cell 2006, 127 (4), 831-846.

3. Fadeel, B.; Xue, D. The ins and outs of phospholipid asymmetry in the plasma membrane: roles in health and disease. Crit. Rev. Biochem. Mol. Biol. 2009, 44 (5), 264-277.

4. Pomorski, T.; Menon, A. K. Lipid flippases and their biological functions. Cellular and Molecular Life Sciences CMLS 2006, 63 (24), 2908-2921.

5. Sebastian, T. T.; Baldridge, R. D.; Xu, P.; Graham, T. R. Phospholipid flippases: building asymmetric membranes and transport vesicles. Biochim. Biophys. Acta 2012, 1821 (8), 1068-1077.

6. Olkkonen, V. M.; Beaslas, O.; Nissila, E. Oxysterols and their cellular effectors. Biomolecules 2012, 2 (1), 76-103.

7. Russell, D. W. Oxysterol biosynthetic enzymes. Biochim. Biophys. Acta 2000, 1529 (1-3), 126-135.

8. Joseph, S. B.; Castrillo, A.; Laffitte, B. A.; Mangelsdorf, D. J.; Tontonoz, P. Reciprocal regulation of inflammation and lipid metabolism by liver $\mathrm{X}$ receptors. Nat. Med. 2003, 9 (2), 213-219. 
9. Kim, S.-M.; Lee, C. W.; Kim, B.-Y.; Jung, Y.-S.; Eo, S.-K.; Park, Y. C.; Kim, K. 27Oxygenated cholesterol induces expression of CXCL8 in macrophages via NF-kappa B and CD88. Biochem. Biophys. Res. Commun. 2015, 463 (4), 1152-1158.

10. Brown, A. J.; Jessup, W. Oxysterols and atherosclerosis. Atherosclerosis 1999, 142 (1), 1-28.

11. Panini, S. R.; Sinensky, M. S. Mechanisms of oxysterol-induced apoptosis. Curr. Opin. Lipidol. 2001, 12 (5), 529-533.

12. Luchetti, F.; Canonico, B.; Cesarini, E.; Betti, M.; Galluzzi, L.; Galli, L.; Tippins, J.; Zerbinati, C.; Papa, S.; Iuliano, L. 7-Ketocholesterol and 5,6-secosterol induce human endothelial cell dysfunction by differential mechanisms. Steroids 2015, 99, 204-211.

13. Smondyrev, A. M.; Berkowitz, M. L. Effects of oxygenated sterol on phospholipid bilayer properties: a molecular dynamics simulation. Chem. Phys. Lipids 2001, 112 (1), 31-39.

14. Kulig, W.; Olzynska, A.; Jurkiewicz, P.; Kantola, A. M.; Komulainen, S.; Manna, M.; Pourmousa, M.; Vazdar, M.; Cwiklik, L.; Rog, T.; Khelashvili, G.; Harries, D.; Telkki, V. V.; Hof, M.; Vattulainen, I.; Jungwirth, P. Cholesterol under oxidative stress-How lipid membranes sense oxidation as cholesterol is being replaced by oxysterols. Free Radical Biol. Med. 2015, 84, 30-41.

15. Olsen, B. N.; Schlesinger, P. H.; Baker, N. A. Perturbations of Membrane Structure by Cholesterol and Cholesterol Derivatives Are Determined by Sterol Orientation. J. Am. Chem. Soc. 2009, 131 (13), 4854-4865.

16. Meaney, S.; Bodin, K.; Diczfalusy, U.; Bjorkhem, I. On the rate of translocation in vitro and kinetics in vivo of the major oxysterols in human circulation: critical importance of the position of the oxygen function. J. Lipid Res. 2002, 43 (12), 21302135.

17. Schroepfer, G. J. Oxysterols: Modulators of cholesterol metabolism and other processes. Physiol. Rev. 2000, 80 (1), 361-554.

18. Leonarduzzi, G.; Gargiulo, S.; Gamba, P.; Testa, G.; Sottero, B.; Rossin, D.; Staurenghi, E.; Poli, G. Modulation of cell signaling pathways by oxysterols in agerelated human diseases. Free Radical Biol. Med. 2014, 75, S5-S5.

19. Wang, P. Y.; Weng, F.; Anderson, R. G. W. OSBP is a cholesterol-regrulated scaffolding protein in control of ERK1/2 activation. Science 2005, 307 (5714), 14721476.

20. Vurusaner, B.; Gamba, P.; Testa, G.; Gargiulo, S.; Biasi, F.; Zerbinati, C.; Iuliano, L.; Leonarduzzi, G.; Basaga, H.; Poli, G. Survival signaling elicited by $27-$

hydroxycholesterol through the combined modulation of cellular redox state and ERK/Akt phosphorylation. Free Radical Biol. Med. 2014, 77, 376-385.

21. Tall, A. R.; Costet, P.; Wang, N. Regulation and mechanisms of macrophage cholesterol efflux. J. Clin. Invest. 2002, 110 (7), 899-904.

22. Wolf, G. The role of oxysterols in cholesterol homeostasis. Nutr. Rev. 1999, 57 (6), 196-8.

23. Bjorkhem, I. Do oxysterols control cholesterol homeostasis? J. Clin. Invest. 2002, $110(6), 725-730$.

24. Bielska, A. A.; Olsen, B. N.; Gale, S. E.; Mydock-McGrane, L.; Krishnan, K.; Baker, N. A.; Schlesinger, P. H.; Covey, D. F.; Ory, D. S. Side-chain oxysterols modulate cholesterol accessibility through membrane remodeling. Biochemistry 2014, 53 (18), 3042-3051. 
25. Olsen, B. N.; Schlesinger, P. H.; Ory, D. S.; Baker, N. A. Side-chain oxysterols: from cells to membranes to molecules. Biochimica et Biophysica Acta (BBA)Biomembranes 2012, 1818 (2), 330-336.

26. Olkkonen, V. M.; Hynynen, R. Interactions of oxysterols with membranes and proteins. Mol. Aspects Med. 2009, 30 (3), 123-133.

27. Torrie, G. M.; Valleau, J. P. Nonphysical sampling distributions in Monte Carlo free-energy estimation: Umbrella sampling. J. Comput. Phys. 1977, 23 (2), 187-199.

28. Berendsen, H. J.; van der Spoel, D.; van Drunen, R. GROMACS: a messagepassing parallel molecular dynamics implementation. Comput. Phys. Commun. 1995, 91 (1-3), 43-56.

29. Hess, B.; Kutzner, C.; van der Spoel, D.; Lindahl, E. GROMACS 4: Algorithms for highly efficient, load-balanced, and scalable molecular simulation. J. Chem. Theory Comput. 2008, 4 (3), 435-447.

30. Maciejewski, A.; Pasenkiewicz-Gierula, M.; Cramariuc, O.; Vattulainen, I.; Rog, T. Refined OPLS All-Atom Force Field for Saturated Phosphatidylcholine Bilayers at Full Hydration. J. Phys. Chem. B 2014, 118 (17), 4571-4581.

31. Kulig, W.; Pasenkiewicz-Gierula, M.; Rog, T. Topologies, structures and parameter files for lipid simulations in GROMACS with the OPLS-aa force field: DPPC, POPC, DOPC, PEPC, and cholesterol. Data in brief 2015, 5, 333-6.

32. Bennett, W. D.; MacCallum, J. L.; Hinner, M. J.; Marrink, S. J.; Tieleman, D. P. Molecular view of cholesterol flip-flop and chemical potential in different membrane environments. J. Am. Chem. Soc. 2009, 131 (35), 12714-12720.

33. Parisio, G.; Sperotto, M. M.; Ferrarini, A. Flip-Flop of Steroids in Phospholipid Bilayers: Effects of the Chemical Structure on Transbilayer Diffusion. J. Am. Chem. Soc. 2012, 134 (29), 12198-12208.

34. Jo, S.; Rui, H.; Lim, J. B.; Klauda, J. B.; Im, W. Cholesterol flip-flop: insights from free energy simulation studies. The Journal of Physical Chemistry B 2010, 114 (42), $13342-13348$.

35. Theunissen, J. J. H.; Jackson, R. L.; Kempen, H. J. M.; Demel, R. A. Membrane properties of oxysterols. Interfacial orientation, influence on membrane permeability and redistribution between membranes. Biochim. Biophys. Acta 1986, 860 (1), 66-74.

36. Subczynski, W. K.; Hyde, J. S.; Kusumi, A. OXYGEN PERMEABILITY OF PHOSPHATIDYLCHOLINE CHOLESTEROL MEMBRANES. Proc. NatI. Acad. Sci. U. S. A. 1989, 86 (12), 4474-4478.

37. Subczynski, W. K.; Wisniewska, A.; Yin, J. J.; Hyde, J. S.; Kusumi, A. Hydrophobic barriers of lipid bilayer - Membranes formed by reduction of water penetration by alkyl chain unsaturation and cholesterol. Biochemistry 1994, 33 (24), 7670-7681. 38. Xiang, T.-X.; Anderson, B. D. Phospholipid surface density determines the partitioning and permeability of acetic acid in DMPC: cholesterol bilayers. J. Membr. Biol. 1995, 148 (2), 157-167.

39. Xiang, T. X.; Anderson, B. D. Phase structures of binary lipid bilayers as revealed by permeability of small molecules. Biochimica Et Biophysica Acta-Biomembranes 1998, 1370 (1), 64-76.

40. Mathai, J. C.; Tristram-Nagle, S.; Nagle, J. F.; Zeidel, M. L. Structural Determinants of Water Permeability through the Lipid Membrane. The Journal of General Physiology 2008, 131 (1), 69-76. 
41. Marsh, D. Polarity and permeation profiles in lipid membranes. Proceedings of the National Academy of Sciences 2001, 98 (14), 7777-7782.

42. Shinoda, W. Permeability across lipid membranes. Biochim. Biophys. Acta 2016, 1858 (10), 2254-2265.

43. Dotson, R. J.; Smith, C. R.; Bueche, K.; Angles, G.; Pias, S. C. Influence of Cholesterol on the Oxygen Permeability of Membranes: Insight from Atomistic Simulations. Biophys. J. 2017, 112 (11), 2336-2347.

44. Anglin, T. C.; Conboy, J. C. Kinetics and thermodynamics of flip-flop in binary phospholipid membranes measured by sum-frequency vibrational spectroscopy. Biochemistry 2009, 48 (43), 10220-10234.

45. Bruckner, R.; Mansy, S.; Ricardo, A.; Mahadevan, L.; Szostak, J. Flip-flopinduced relaxation of bending energy: implications for membrane remodeling. Biophys. J. 2009, 97 (12), 3113-3122.

46. Sapay, N.; Bennett, W. D.; Tieleman, D. P. Thermodynamics of flip-flop and desorption for a systematic series of phosphatidylcholine lipids. Soft Matter 2009, 5 (17), 3295-3302.

47. Lange, Y.; Ye, J.; Strebel, F. MOVEMENT OF 25-HYDROXYCHOLESTEROL FROM THE PLASMA-MEMBRANE TO THE ROUGH ENDOPLASMIC-RETICULUM IN CULTURED HEPATOMA-CELLS. J. Lipid Res. 1995, 36 (5), 1092-1097.

48. Lange, Y.; Ye, J.; Rigney, M.; Steck, T. L. Regulation of endoplasmic reticulum cholesterol by plasma membrane cholesterol. J. Lipid Res. 1999, 40 (12), 2264-2270. 